\title{
UNIVERSAL SAMPLING OF SIGNALS WITH FINITE RATE OF INNOVATION
}

\author{
Xiaoyao Wei and Pier Luigi Dragotti \\ Electrical and Electronic Engineering Department \\ Imperial College London \\ Email: ivy.wei@imperial.ac.uk,p.dragotti@imperial.ac.uk
}

\begin{abstract}
Recently it has been shown that specific classes of nonbandlimited signals known as signals with finite rate of innovation (FRI) can be perfectly reconstructed by using appropriate sampling kernels and reconstruction schemes. This exact FRI framework was later extended to an approximate FRI framework that works with any kernel.

Reconstruction is achieved by recovering all the parameters in the parametric model of the incoming signal, hence it is essential to know the model order (the rate of innovation) to ensure recovery. In view of this, we devise an algorithm for identifying the rate of innovation in order to extend the current sampling scheme to a universal one which enables sampling signals with arbitrary FRI using any acquisition device. Our proposed algorithm can effectively identify the rate of innovation prior to the signal reconstruction using arbitrary kernels and in different noise levels where we also show that it achieves the performance predicted by the Cramèr-Rao bounds.
\end{abstract}

\section{INTRODUCTION}

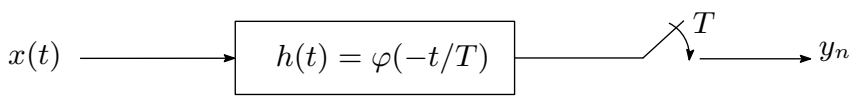

Fig. 1. A typical sampling set-up.

In a typical sampling set-up as shown in Fig. 1, the input analog signal $x(t)$ is filtered through $h(t)$ which is an antialiasing filter, then the filtered input signal $y_{s}(t)=h(t) *$ $x(t)$ goes through an analog-to-digital converter (ADC) with a sampling rate $1 / T$ and outputs samples $y_{n}$, which are given by $y_{n}=\langle x(t), \varphi(t / T-n)\rangle$, where $\varphi(t)=h(-t T)$ is the sampling kernel. From the set of samples $y_{n}$ we want to recover $x(t)$ perfectly and uniquely.

Recently it has been shown that it is possible to develop sampling schemes for classes of signals that are neither bandlimited nor belong to a fixed subspace, but are completely

This work is supported by the European Research Council (ERC) starting investigator award Nr. 277800 (RecoSamp). specified by a finite number of free parameters per unit of time and are called signals with finite rate of innovation $[1,2,3]$. Example of such signals include streams of Diracs, streams of pulses [4], piecewise sinusoidals [5] and planar polygons [6].

In traditional FRI, the sampling kernel cannot be arbitrary but needs to satisfy certain properties that depend on the rate of innovation of the analogue signal. For example, if the incoming signal is a stream of Diracs with at most $K$ Diracs per unit of time, the rate of innovation is $\rho=2 K$ and the kernel is designed so that any stream of Diracs with $\rho \leq 2 \mathrm{~K}$ can be reconstructed. However normally the same kernel cannot reconstruct signals with $\rho>2 K$ even if we increase the sampling rate.

In this paper, we use and extend the results in [7] in order to devise a method for sampling streams of Diracs with unknown rate of innovation and using arbitrary kernels. We show numerically that in close to noiseless setups we can retrieve $K$ Diracs per unit of time when the sampling rate is $1 / T \geq c \cdot 2 K$, where we find $c=1.6$ empirically. This is achieved by first estimating the rate of innovation of the signal and then reconstructing it. If $\rho>1 / T$ then reliable reconstruction is achieved by increasing the sampling rate but crucially without the need of changing the kernel. The algorithm is also effective in noisy scenarios where we show that it achieves the performance predicted by the Cramér-Rao bounds.

The paper is organised as follows: in Section 2 we provide an overview of the theory of sampling signals with FRI, we show that streams of Diracs can be perfectly reconstructed with specific sampling kernels. We then present in Section 3 the approximate framework and how we use it together with our proposed algorithm to enable universal sampling of signals with arbitrary FRI and using arbitrary kernels. Simulation results are shown in Section 4. Finally we conclude in Section 5 .

\section{OVERVIEW OF FINITE RATE OF INNOVATION THEORY}

Consider the sampling set-up in Fig. 1. We introduce a specific class of sampling kernels that allows perfect recovery of 
$x(t)$ from the samples $y_{n}$. This is the family of exponential reproducing functions where any family member $\varphi(t)$ together with its shifted versions can reproduce complex exponentials:

$$
\sum_{n \in \mathbb{Z}} c_{m, n} \varphi(t-n)=\mathrm{e}^{\alpha_{m} t}, \quad m=0,1, \ldots, M
$$

for proper coefficients $c_{m, n}$. It is possible to show that a function satisfies (1) if and only if it meets the generalised StrangFix conditions:

$$
\hat{\varphi}\left(\alpha_{m}\right) \neq 0 \text { and } \hat{\varphi}\left(\alpha_{m}+j 2 \pi l\right)=0 \quad l \in \mathbb{Z} \backslash\{0\}
$$

where $\hat{\varphi}(s)$ is the bilateral Laplace transform of $\varphi(t)$.

Note that the exponential reproducing kernels most robust to noise are called exponential-MOMS (e-MOMS) and where introduced in [8].

An important characteristic of the exponential reproducing kernel is that it allows us to map the samples $y_{n}$ with the Laplace or Fourier transform of $x(t)$ at $\left\{\alpha_{m}\right\}_{m=0}^{M}$ and this independently of the input signal. Assume that the signal $x(t)$ is of compact support such that it is characterised by only $N$ non-zero samples. Consider the following weighted sum of these samples, where the weights $c_{m, n}$ are those in (1) that reproduce $\mathrm{e}^{\alpha_{m} t}$ :

$$
\begin{aligned}
s_{m} & =\sum_{n} c_{m, n} y_{n} \\
& =\left\langle x(t), \sum_{n} c_{m, n} \varphi(t-n)\right\rangle \\
& =\int_{-\infty}^{\infty} x(t) \mathrm{e}^{\alpha_{m} t} d t, \quad m=0,1, \ldots, M .
\end{aligned}
$$

Note that $\int_{-\infty}^{\infty} x(t) \mathrm{e}^{\alpha_{m} t} d t$ is exactly the bilateral Laplace transform of $x(t)$ evaluated at $\left\{\alpha_{m}\right\}_{m=0}^{M}$ and denoted by $\hat{x}\left(\alpha_{m}\right)$. Moreover, when $\alpha_{m}$ is purely imaginary, $\hat{x}\left(j \omega_{m}\right)$ is the Fourier transform of $x(t)$ at $\omega=\omega_{m}$.

When $x(t)$ is a specific class of signals with FRI and $\alpha_{m}$ is chosen to be of the form $\alpha_{m}=\alpha_{0}+m \lambda$, it is possible to establish a one-to-one mapping between $\hat{x}\left(\alpha_{m}\right)$ and $x(t)$. For example, if $x(t)=\sum_{k=0}^{K-1} a_{k} \delta\left(t-t_{k}\right)$ is a stream of $K$ Diracs located at $t_{k}$ then the weighted sum of the samples

$$
\begin{aligned}
s_{m} & =\sum_{n} c_{m, n} y_{n} \\
& =\int_{-\infty}^{\infty} \sum_{k=0}^{K-1} a_{k} \delta\left(t-t_{k}\right) \mathrm{e}^{\alpha_{m} t} d t \\
& =\sum_{k=0}^{K} \hat{a}_{k} u_{k}^{m}, \quad m=0,1, \ldots, M,
\end{aligned}
$$

is a sum of exponentials, where $\hat{a}_{k}=a_{k} \mathrm{e}^{\alpha_{0} t_{k}}$ and $u_{k}=\mathrm{e}^{\lambda t_{k}}$. Retrieving $\left\{\hat{a}_{k}, u_{k}\right\}_{k=0}^{K-1}$ from $\left\{s_{m}\right\}_{m=0}^{M}$ is a classical problem in spectral estimation and can be solved by Prony's method (annihilating filter method [1, 3]). For noisy FRI signal retrieval, Cadzow method [9] and matrix pencil [10] is proven to be effective.

We also note that this formulation requires the acquisition device to behave like exponential reproducing function and its order must be equal to or larger than the rate of innovation of the signal with FRI, specifically, for this example $M \geq$ $2 K-1$. This means that if the incoming signal has more than $K$ Diracs, e.g. $K^{\prime}>K$, it cannot be reconstructed with this kernel and this even when $N \geq 2 K^{\prime}$.

In the next section we show how to overcome this limitation.

\section{UNIVERSAL SAMPLING OF SIGNALS WITH FINITE RATE OF INNOVATION}

\subsection{FRI Sampling using Arbitrary Kernels}

In the previous section, we have shown that the reconstruction of FRI signals with specific rate of innovation is dependent on proper design of the acquisition devices. Recently, the FRI sampling theory has been extended so that any acquisition device can be used [7].

Consider an arbitrary kernel $\varphi(t)$. We want to find a linear combination of $\varphi(t)$ with its shifted versions that provides the best approximation to a specific exponential, more specifically, find coefficients $c_{n}$ such that:

$$
\sum_{n \in \mathbb{Z}} c_{n} \varphi(t-n) \approx \mathrm{e}^{\alpha t} .
$$

This approximation is exact only when the kernel $\varphi(t)$ satisfied the generalized Strang-Fix condition. For any other function, the coefficients $c_{n}$ that best fit (5) are desired.

For the sake of clarity, we use $c_{n}=c_{0} \mathrm{e}^{\alpha n}$ and then we can show that the error in approximating the exponential is:

$$
\epsilon_{\text {approx }}(t)=\mathrm{e}^{\alpha t}\left[1-c_{0} \sum_{l \in \mathbb{Z}} \hat{\varphi}(\alpha+j 2 \pi l) \mathrm{e}^{j 2 \pi l t}\right] .
$$

Note that if the Laplace transform of $\varphi(t)$ decays sufficiently quickly, we can assume the terms $\hat{\varphi}(\alpha+j 2 \pi l)$ are close to zero for $l \in \mathbb{Z} \backslash\{0\}$. In this case, the approximation error is minimised when $c_{n}=\hat{\varphi}(\alpha)^{-1} \mathrm{e}^{\alpha n}$, requiring only the knowledge of $\varphi(t)$ at $\alpha$.

Recall that in the exact reproduction framework, the number of exponentials we can reproduce is dependent on the order of the sampling kernel and that an acquisition device may be no longer usable when the rate of innovation of an incoming signal exceeds the kernel's order. In contrast, in [7] we notice that for the approximate framework, $N$ samples can give us $N$ approximate exponentials and this directly relates the highest rate of innovation it can recover to the sampling rate rather than the order of the kernel. Hence any acquisition device is always usable for signals with arbitrary rate of innovation below the sampling rate. 


\subsection{Identification of the Rate of Innovation}

We have shown that the approximate FRI theory allows us to sample FRI signals using any sampling kernel. Together with the algorithm we are going to propose for identifying the rate of innovation, we will extend the current sampling scheme to a universal one which can recover signals with arbitrarily unknown finite rate of innovation using any sampling kernel.

The general idea behind our algorithm is as follows. Given $c N(c>1)$ samples of the input stream of Diracs $y_{n}$, we are able to obtain $c N$ approximated Fourier coefficients $\hat{x}\left(\alpha_{m}\right), m=1, \ldots, c N$. From these coefficients we estimate at most $N / 2$ number of Diracs. Note that theoretically $N$ samples is enough for recovering $N / 2$ Diracs, but in reality we require a slightly higher number of samples per unit time since the Fourier coefficients are all approximated.

We first assume that the number of the Diracs is $p=1$ and we retrieve the location and amplitude of the Dirac in the parametric model $\sum_{k=1}^{p} a_{k} \delta\left(t-t_{k}\right)$. Next we resynthesize the samples and compute the error on the resynthesized samples with respect to $y_{n}$. Then we repeat this procedure but with assumption that $p$ is 2,3 up to $N / 2$.

We expect that the error on the samples will first decrease gradually when the number of Diracs $p$ we assumed approach the true number $K$ and will eventually reach nearly zero when $p$ is exactly the number of the Diracs. When we further increase $p$, the errors will either rise slightly or further decrease with a much slower rate. In either case, the turning point can be recognised from the second derivative of the error. Once the number of Diracs $K$ is known, the input signal $x(t)$ can be recovered using the parametric model with correct order.

We summarize the algorithm as follows:

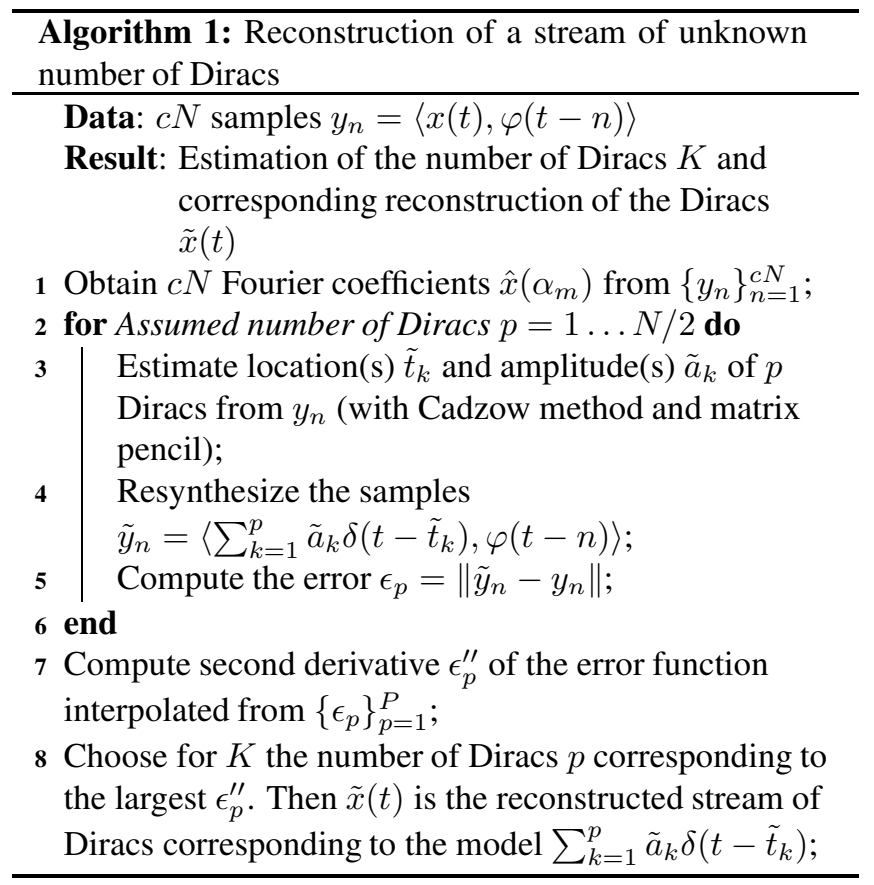

\section{SIMULATIONS}

\subsection{Universal Sampling in the Absence of Noise}

In this section, we show that our proposed algorithm is universal in that any acquisition device can be used for sampling and any unknown number $(K)$ of Diracs can be recovered almost perfectly with a sampling rate $1 / T \geq 2 c K$, where $c=1.6$ in our simulations.

Assume we have a stream of unknown number $K$ of Diracs and we take $c N$ samples with a B-spline of order 5 following the scheme in Fig. 1. In the exact framework, this specific acquisition device restricts the number of Diracs we can reconstruct to 3. Thanks to the approximate StrangFix framework, with a B-spline of order 5 we can build $c N$ approximated Fourier coefficients which allows us to reconstruct 1 up to $N / 2$ Diracs. In Fig. 2(a,b) we show that by using our proposed algorithm the number of Diracs $K=31$ is identified from $c N=99$ samples, and then all the 31 Diracs are almost perfectly reconstructed in the absence of noise.

We also highlight the universality of the sampling scheme that even if the input streams of numbers of Diracs changes, for example in Fig. 2(c,d) where $K$ changes from 31 to 21, reliable reconstruction can still be achieved without the need of changing the set-up.

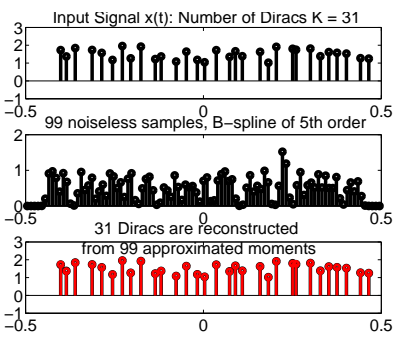

(a) 31 Diracs are reconstructed from 99 samples in noiseless case.

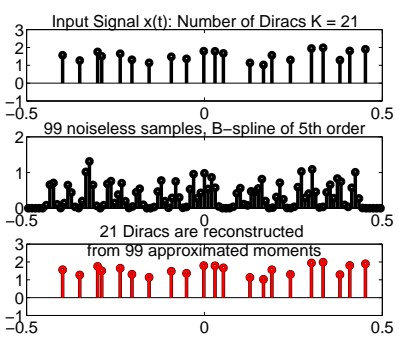

(c) 21 Diracs are reconstructed from 99 samples in noiseless case.

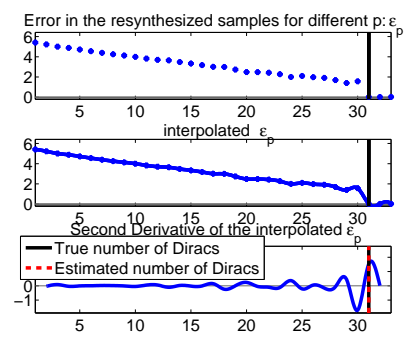

(b) $K=31$ is identified.

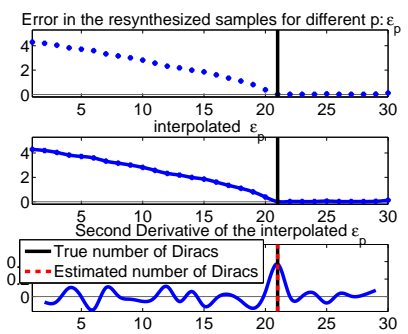

(d) $K=21$ is identified.
Fig. 2. Universal sampling of a stream of unknown number of Diracs using B-spline kernel of order 5 in the absence of noise. (b)(d) The number of Diracs is identified from second derivative of the error function. (a)(c) All the Diracs are retrieved almost perfectly. 


\subsection{Universal Sampling in the Presence of Noise}

In the following simulations we show that the algorithm is also effective in noisy scenario since it achieves the performance given by the moment-based Cramér-Rao bounds [7]. Here the noise is added to the samples $y_{n}$ and is white Gaussian noise of variance $\sigma$, chosen according to the target signalto-noise ratio defined as $\operatorname{SNR}(\mathrm{dB})=10 \log \frac{\|\mathbf{y}\|^{2}}{N \sigma^{2}}$. Note that in this sampling scheme, a larger sampling rate corresponds to more robust reconstruction, so in the noisy scenario we use a sampling rate $1 / T$ larger than what we used in the noiseless case, i.e. $1 / T=2 c^{\prime} K>2 c K$.

We now compute the Cramér-Rao bound for the situation where there are two Diracs with same amplitude sampled at the rate $1 / T=31$. Fig. 3(a) shows that the observed standard deviation given by the FRI reconstruction algorithm in general reaches the theoretical minimum given by Cramér-Rao bounds for distances $d$ beyond the critical values, which are the intersects of the bounds and the line $d=2 \times 3 \sigma_{\mathrm{CRB}}$ in Fig. 3(a).

For distances smaller than the critical values, it is possible that these two Diracs are indistinguishable and the FRI reconstruction algorithm reconstruct them as one tall Dirac situated in between the true Diracs and one Dirac far away from the true Diracs with negligible amplitude. This is not surprising. We can see from Fig. 3(b) that when the two Diracs get closer, the uncertainty $\sigma_{\mathrm{CRB}}$ on the locations increases. When the distance reaches the critical value $2 \times 3 \sigma_{\mathrm{CRB}}$, in which case the "three-sigma" uncertainty on the location of the first Dirac overlaps that of the second one, the two Diracs are possibly indistinguishable. In this situation, our algorithm for identifying the number of Diracs, which is based on the FRI reconstruction algorithm, will neglect the one with negligible amplitude and identify only one Dirac.
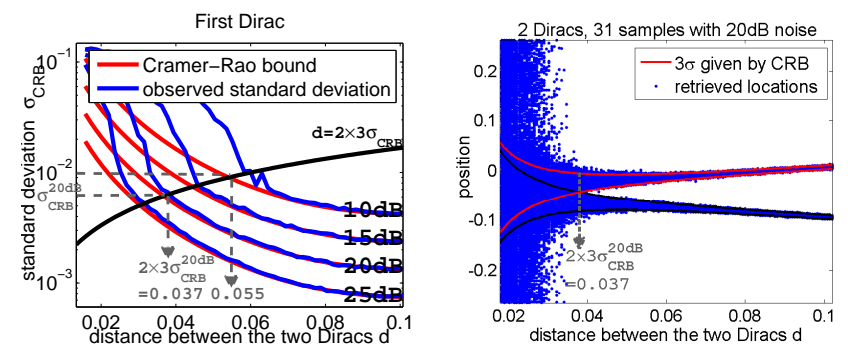

(a) FRI algorithm reaches CRB for (b) scatterplot of the locations in distances beyond the critical values. $20 \mathrm{~dB}$ noise.

Fig. 3. (a) observed standard deviation compared to CramérRao lower bounds on the location of one of the two Diracs. (2000 realisations for each distance) (b) scatterplot of retrieved locations compared to 3 times the standard deviation given by Cramér-Rao lower bounds.

The results in Fig. 4 are also consistent with our observation regarding Cramér-Rao bounds in Fig. 3. In this exam- ple, there are 8 Diracs sampled at $1 / T=31$, in which two Diracs are close to each other and the others are well separated. We notice that all the 8 Diracs are accurately reconstructed in $20 \mathrm{~dB}$ noise. In $10 \mathrm{~dB}$ noise, however, the algorithm recognises the number of Diracs as $K=7$ and the two close Diracs, whose distance, 0.046 , is smaller than the critical distance 0.055 given by the Cramér-Rao bounds, as one Dirac. Nevertheless, we highlight that this can be easily solved by increasing the sampling rate where the Cramér-Rao bound and the critical distance will decrease.
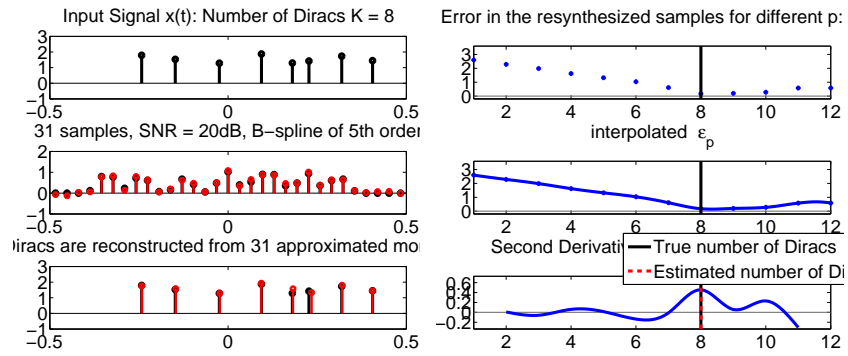

(a) 8 Diracs are reconstructed in $20 \mathrm{~dB}$ noise.

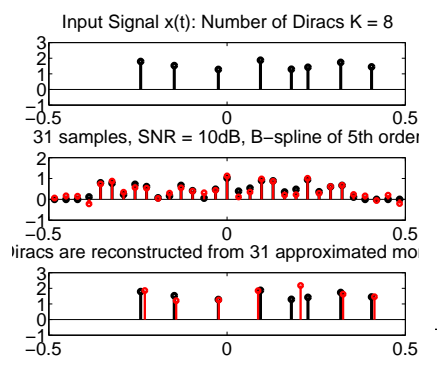
Error in the resynthesized samples for different $\mathrm{p}$ :

(c) 7 Diracs are reconstructed in 10dB noise.

(b) Estimate the number of Diracs
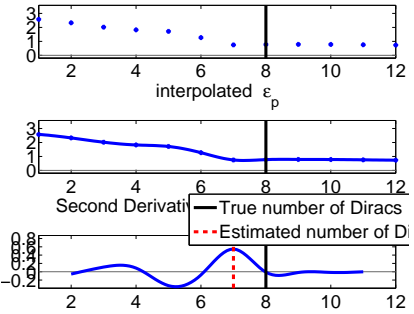

(d) Estimate the number of Diracs

Fig. 4. Universal sampling of a stream of unknown number of Diracs using B-spline kernel of order 5 in the presence of noise. Two close Diracs in the 8 Diracs are recognised as one Dirac in $10 \mathrm{~dB}$ noise and the others are accurately retrieved.

To conclude, our proposed algorithm is able to identify the model order correctly and is consequently able to reconstruct all the stream of Diracs almost perfectly in the absence of noise. In the noisy situation the algorithm achieved the best possible result as indicated by the Cramér-Rao bounds.

\section{CONCLUSION}

In this paper we have shown how to sample FRI signals with arbitrary kernels and that a novel algorithm can identify the model order accurately prior to reconstruction. Simulation results have confirmed the effectiveness of the proposed method. 


\section{REFERENCES}

[1] Martin Vetterli, Pina Marziliano, and Thierry Blu, "Sampling Signals with Finite Rate of Innovation," IEEE Transactions on Signal Processing, vol. 50, no. 6, pp. 1417-1428, 2002.

[2] Thierry Blu, Pier Luigi Dragotti, Martin Vetterli, Pina Marziliano, and Lionel Coulot, "Sparse Sampling of Signal Innovations," IEEE Signal Processing Magazine, vol. 25, no. 2, pp. 31-40, 2008.

[3] Pier Luigi Dragotti, Martin Vetterli, and Thierry Blu, "Sampling Moments and Reconstructing Signals of Finite Rate of Innovation: Shannon Meets Strang-Fix," IEEE Transactions on Signal Processing, vol. 55, no. 5, Part 1, pp. 1741-1757, 2007.

[4] Ronen Tur, Yonina C Eldar, and Zvi Friedman, "Innovation Rate Sampling of Pulse Streams with Application to Ultrasound Imaging," IEEE Transactions on Signal Processing, vol. 59, no. 4, pp. 1827-1842, 2011.

[5] Jesse Berent, Pier Luigi Dragotti, and Thierry Blu, "Sampling Piecewise Sinusoidal Signals with Finite Rate of Innovation Methods," IEEE Transactions on Signal Processing, vol. 58, no. 2, pp. 613-625, 2010.

[6] Pancham Shukla and Pier Luigi Dragotti, "Sampling Schemes for Multidimensional Signals with Finite Rate of Innovation," IEEE Transactions on Signal Processing, vol. 55, no. 7, pp. 3670-3686, 2007.

[7] Jose Antonio Urigüen, Thierry Blu, and Pier Luigi Dragotti, "FRI sampling with arbitrary kernels," IEEE Transactions on Signal Processing, vol. 61, no. 21, pp. 5310-5323, 2013.

[8] Jose Antonio Urigüen, Pier Luigi Dragotti, and Thierry Blu, "On the Exponential Reproducing Kernels for Sampling Signals with Finite Rate of Innovation," Proceedings of Sampling Theory and Applications (SampTA), 2011.

[9] James A Cadzow, "Signal Enhancement-A Composite Property Mapping Algorithm," IEEE Transactions on Acoustics, Speech and Signal Processing, vol. 36, no. 1, pp. 49-62, 1988.

[10] Yingbo Hua and Tapan K Sarkar, "Matrix Pencil Method for Estimating Parameters of Exponentially Damped/Undamped Sinusoids in Noise," IEEE Transactions on Acoustics, Speech and Signal Processing, vol. 38, no. 5, pp. 814-824, 1990. 